Pandemia, jóvenes y precarización laboral

Repertorios y acciones colectivas de les trabajadores de plataformas en CABA

Dylan Braian Cruz Tolosa

Revista Argentina de Estudios de Juventud, (14), e051, 2020

ISSN 1852-4907 | https://doi.org/10.24215/18524907e051

https://perio.unlp.edu.ar/ojs/index.php/revistadejuventud

FPyCS | Universidad Nacional de La Plata

La Plata | Buenos Aires | Argentina

\title{
PANDEMIA, JÓVENES \\ Y PRECARIZACIÓN LABORAL
}

\section{REPERTORIOS Y ACCIONES COLECTIVAS \\ DE LES TRABAJADORES DE PLATAFORMAS EN CABA}

\author{
Pandemic, Youth and Job Insecurity \\ Repertoires and Collective Actions of the Workers of the Platforms in CABA
}

\author{
Dylan Braian Cruz Tolosa \\ dylan98tolosa@gmail.com \\ https://orcid.org/0000-0002-8256-7953
}

Consejo Interuniversitario Nacional (CIN)

Facultad de Ciencias Humanas

Universidad Nacional del Centro de la Provincia de Buenos Aires (UNICEN)

Argentina

Palabras clave

jóvenes

precarización laboral pandemia acciones colectivas

Keywords

young people job insecurity pandemic collective actions

\section{Resumen}

En el presente trabajo se ofrecen aproximaciones a las condiciones contractuales, materiales y subjetivas que atraviesan el cotidiano de les jóvenes en el marco de sus jornadas laborales en las plataformas digitales en la Ciudad Autónoma de Buenos Aires (CABA), Argentina. En este sentido, se abordan los procesos de precarización laboral y se identifican los impactos y las consecuencias objetivas y subjetivas en les trabajadores, la conformación de identidades, las potencialidades de la organización colectiva y los desafíos actuales de una actividad que, en el contexto de la Pandemia por COVID-19, fue declarada esencial en todo el mundo.

\section{Abstract}

This work offers approaches to the contractual, material and subjective conditions that go through the daily lives of young people within the framework of their working hours on digital platforms in the Ciudad Autónoma de Buenos Aires (CABA), Argentina. In this sense, it is proposed to address the processes of job insecurity by identifying the impacts and the objective and subjective consequences on workers, the conformation of identities, the potentialities of the collective organization and the current challenges of an activity which, in the context of the Pandemic COVID-19, was declared essential around the world.

Recibido 05/09/2020 Aceptado 17/09/2020 Publicado 16/10/2020 


\title{
PANDEMIA, JÓVENES \\ Y PRECARIZACIÓN LABORAL
}

\author{
Repertorios y acciones colectivas \\ de les trabajadores de plataformas en CABA
}

\section{Por Dylan Braian Cruz Tolosa}

La calle sigue siendo el escenario de la política y de las grandes transformaciones del país.

Cristina Fernández de Kirchner (2018)

\section{Introducción}

El presente trabajo ${ }^{1}$ pretende contribuir al análisis de la precariedad laboral juvenil en una Argentina que, igualando la realidad de muchos países del mundo, se encuentra atravesando un contexto pandémico que ocupa una centralidad en la agenda de los organismos gubernamentales, principalmente de aquellos que atañen al sistema de salud público en sus escalas nacionales, provinciales y municipales, con el objetivo de evitar contagios mediante las medidas de prevención que se impulsan en el marco del Aislamiento Social Preventivo y Obligatorio (ASPO) ${ }^{2}$ para garantizar la atención sanitaria frente a la masividad de contagios que se registran a diario y para preservar la vida como decisión ética, moral y política de la gestión a cargo del Estado nacional.

Desde una perspectiva de la sociología del trabajo, se propone abordar las condiciones laborales de les $^{3}$ jóvenes, tanto las particularidades como heterogeneidades presentes en el grupo social, a partir de aproximaciones a las relaciones laborales de les trabajadores de las aplicaciones y/o plataformas en la Ciudad Autónoma de Buenos Aires (CABA). En otras palabras, se busca comprender 
los aspectos estructurales, subjetivos y las relaciones de poder y/o resistencias ubicados en un marco sociohistórico que, para los fines analíticos del trabajo, abarca desde finales de 2015 - con la asunción del presidente Mauricio Macri y la instauración de un modelo económico neoliberal- hasta la actualidad y recupera, particularmente, el contexto mundial signado por la pandemia de COVID-19 que implicó reconfiguraciones en todos los ámbitos de la vida, entre ellos, el laboral.

La precarización laboral emerge como concepto en las ciencias sociales en los primeros años de la década de 1970 con el objeto de explicar y de denunciar un proceso de degradación en las condiciones laborales que se instala a partir de las grandes transformaciones globales del trabajo junto con el avance del proyecto neoliberal en la región latinoamericana. Al calor de los cambios estructurales, la precariedad se expandió jurídicamente en nuevas formas laborales que encuentran marcos normativos inestables, flexibles y desprotegidos para les trabajadores.

Frente a ello, les jóvenes, lejos de naturalizar las condiciones de precarización laboral, permiten evidenciar la conformación de un horizonte del «buen empleo» y el despliegue de estrategias y la organización colectiva para la transformación de las condiciones, en tanto sujetos colectivos. La acción colectiva, bajo cualquiera de sus formas y sus modalidades no puede ser comprendida sino por sus relaciones, directas o indirectas, con el Estado, sea en términos de cooperación o de conflicto. En el marco general de la pandemia y de las medidas que el Estado ha tomado en relación con la emergencia sanitaria, cabe preguntarse acerca de las reales posibilidades de acción colectiva, sus límites, sus potencialidades y sus desafíos. El análisis de la movilización y la acción colectiva tiende a complejizarse si se tiene en cuenta que las oportunidades políticas no solo se generan desde una supuesta omnipresencia del Estado sino que se producen también desde las dinámicas colectivas organizacionales.

En términos esquemáticos, el trabajo se estructura en tres apartados. En primer lugar, se introducen los aspectos generales de la economía de las plataformas, recuperando a les jóvenes como una parte significativa de la totalidad de la fuerza de trabajo empleada; un segundo lugar, se recuperan las modificaciones que introduce como así también los procesos de resistencias que generó la aprobación de la Ley 6314 de Regulación de las Tareas Laborales por la Legislatura porteña 
en julio de 2020; por último, se ofrecen las consideraciones finales que, a partir de la recuperación de los repertorios y las acciones colectivas de les propies jóvenes, intentan sintetizar algunos desafíos del mundo laboral a corto y a largo plazo, con el fin de garantizar una construcción de relaciones laborales más justas.

\section{La economía de las plataformas: jóvenes y precarización laboral}

La precariedad laboral es, desde hace tiempo, uno de los principales problemas del mundo laboral. Este proceso remite, en palabras de Diana Menéndez (2010), a un conjunto de dimensiones asociadas al deterioro general de las condiciones de trabajo (ya materiales, ya simbólicas) que afectan a les trabajadores tanto en la Argentina como en el resto del mundo en el marco de un sistema de producción capitalista que presenta cada vez mayores desigualdades. En términos paradójicos, las etapas del desarrollo del capital demuestran que cada vez son menos las personas que concentran la mayor parte de la riqueza, mientras que el resto padece, cada vez más, los sinsabores de las injusticias.

El fenómeno de las plataformas como Uber Eats, Rappi, Glovo o PedidosYA cuenta con aproximadamente diez años a nivel mundial y ha significado una de las mayores disrupciones en el mundo del trabajo.

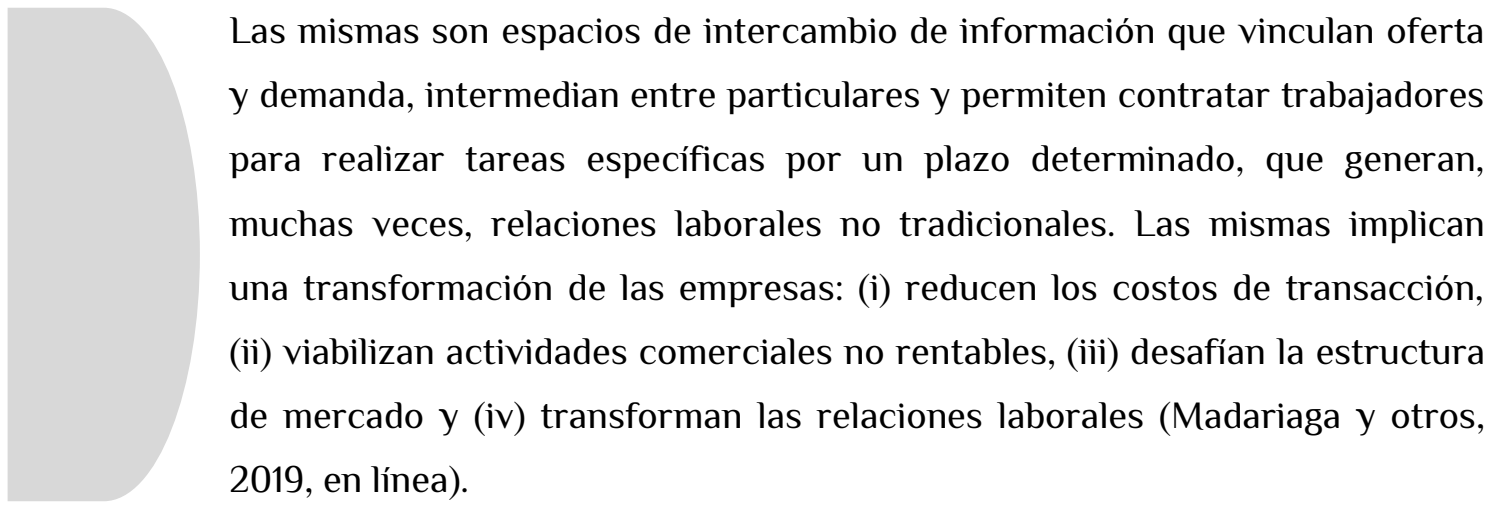

Estas plataformas corresponden a empresas de capital extranjero que ingresaron al país en 2016 producto, entre otros factores, de la flexibilización del sistema de pagos al exterior impulsada por las políticas macroeconómicas del proyecto neoliberal del macrismo. Un informe elaborado por el CIPPEC, ${ }^{4}$ BID LAB y la OIT (Madariaga y otros, 2019) presenta la desvinculación de las relaciones laborales con 
estas empresas respecto de toda regulación laboral; esto conlleva que las formas de trabajo en estas plataformas no sean alcanzadas por las estadísticas oficiales y queden invisibilizadas en categorías ${ }^{5}$ como «ocupados no asalariados», «trabajadores por cuenta propia», entre otras.

De acuerdo a los resultados del informe, les jóvenes trabajadores predominan en los empleos de las plataformas digitales. La totalidad se compone, aproximadamente, por 160.000 trabajadores, de les cuales 60.000 son de reparto. El $90 \%$ no supera los 60 años y un tercio tiene menos de 30 años. En relación con los trabajos para les jóvenes, Julieta Longo y Mariana Busso (2017) sostienen:

[...] los trabajos y los empleos precarios configuran escenarios diversos e incluso antagónicos para los jóvenes que ingresan al mercado de trabajo: posibilitan o restringen la posibilidad de articular actividades laborales con distintas situaciones que nos presentan las esferas familiar, educativa y de ocio, entre otras; y habilitan o impiden la posibilidad de obtener contratos estables y registrados en el mercado de trabajo, en el corto, mediano o largo plazo (p. 9).

Asimismo, existe una marcada disparidad de género, siendo el trabajo en las plataformas altamente masculinizado. Según los datos del informe, 4 de cada 5 trabajadores son hombres. Pablo Pérez (2016) afirma que «muchas mujeres se ven imposibilitadas de participar en el mercado laboral por asumir el cuidado de los niños en el hogar, situación que difiere para los varones y es indicativa de la persistencia del modelo del varón proveedor», al tiempo que señala las «fuertes implicancias sobre las posibilidades laborales de ambos sexos, involucrando menores tasas de participación laboral y empleo para las mujeres como norma social (y no como elección racional)»(p. 48).

Con respecto al nivel educativo, si bien varía sustancialmente según la plataforma, les trabajadores cuentan con niveles de estudios elevados. 90\% finalizó los estudios secundarios y $37 \%$ afirma haber terminado estudios superiores, lo que da cuenta de que el proceso de precarización laboral presente en la generalidad de los trabajos no supone o no implica, necesariamente, pobreza. Longo y Busso (2017) sostienen que la precariedad laboral «se inserta en anclajes estructurales que sobrepasan el marco biográfico de los individuos, y son esos anclajes los que determinan más particularmente la exposición a la pobreza entre las trayectorias precarias» (p. 11). 
Este punto nos convocaría a analizar aspectos en torno a las dificultades que tienen les jóvenes para encontrar un empleo «tradicional» y por lo cual recurren a las plataformas. Sin embargo, es pertinente mencionar que gran parte de les trabajadores que se dedican exclusivamente a las plataformas, en promedio, estuvieron cuatro meses buscando otras oportunidades laborales. La jornada laboral abarcaría hasta 40 horas semanales, con horarios rotativos y coloca expectativas en la posibilidad de aumentar los ingresos. En el caso de las condiciones laborales prima una «cierta» informalidad: según los datos arrojados por el informe, solo $55 \%$ de les encuestades realiza aportes provisionales por su actividad en la plataforma a través del régimen de monotributo y apenas $40 \%$ posee obra social a raíz de su trabajo.

Precariedad y virtualidad son dos claves que nos permiten reconstruir el mundo laboral en los tiempos que corren. En el marco de la pandemia que golpea las realidades económicas, sociales y políticas de todos los países, el gobierno nacional decretó medidas de emergencia y de prevención, siendo el ASPO la de mayor impacto social. El mundo laboral presentó nuevas situaciones que requieren de respuestas específicas; por un lado, porque aparecen escenarios por «fuera de la ley", por el otro, por la necesidad de la formación de trabajadores acompasados a un tiempo histórico determinado por la inmediatez, la fragilidad de la experiencia, la individualidad y la segmentación de la vida común. En esta línea, Lucía De Gennaro (2020) plantea que la regulación laboral para les trabajadores de las plataformas, aprobada el pasado 16 de julio por la Legislatura porteña, se inscribe en ese sentido, al proponer discusiones e intentar dar respuestas para estos tiempos; pero en la «creatividad» de las empresas se busca salirse del juego y de esta manera agudizar quitas de derechos y obligaciones.

\section{La construcción de identidad y los procesos de resistencias}

La pandemia de COVID-19 ha producido efectos políticos, sociales, económicos y culturales que atañen a los ámbitos de gestión estatal. Estos ponen en evidencia las desigualdades preexistentes, que activan decisiones económicas de distinto tipo y alcance, así como también modifican prácticas y comportamientos de la población. 
En este marco, el campo de las acciones colectivas también ha experimentado modificaciones.

El Subsecretario de Trabajo, Industria y Comercio de la Ciudad Autónoma de Buenos Aires, Ezequiel Jarvis, ha ensayado diversas salidas para las nuevas circunstancias. Sin embargo, la iniciativa del oficialismo (UCR-PRO) cosechó rechazos por parte de los bloques opositores y, principalmente, por parte de les propies trabajadores de las plataformas, quienes se movilizaron hasta la Legislatura para expresar su repudio. Examinemos, brevemente, algunos procesos: en octubre de 2018, les trabajadores formaron en nuestro país el primer sindicato del rubro en la región. Para sus integrantes, la Asociación de Personal de Plataformas (APP) «será una forma de darle mayor legitimidad» a sus reclamos (Gestión Sindical, 10/10/2018). Debido a la pandemia por COVID-19, a principios de marzo de 2020, en la Argentina y en el mundo entero, la actividad fue declarada esencial; no obstante, la agudización de la crisis sanitaria incrementó la vulnerabilidad de les trabajadores tanto en la estabilidad como en la calidad de sus condiciones de trabajo. Frente a esto, el 1 de julio de 2020, se llevó adelante el tercer Paro Internacional de Repartidores de Aplicaciones, una de las acciones globales que buscó visibilizar la importancia de la tarea y la fragilidad en la que es desarrollada

El titular de la APP, Roger Rojas, denunció en diferentes medios de comunicación las irregularidades de estas empresas. Entre ellas, apuntó al incumplimiento de derechos laborales básicos, ante situaciones como las jornadas de hasta 16 horas diarias o el hecho de que todos los insumos necesarios para realizar las tareas (vehículos, bicicletas, teléfonos, renta, entre otros) corren por cuenta de les trabajadores. En una nota publicada en el diario Página/12, Rojas señaló que no les conocen las caras. Las empresas utilizan expresiones como «independientes», «propios jefes» o "colaboradores», que lejos están de reconocerles como trabajadores. Por cada fin de semana trabajado, la empresa, en carácter de incentivos, les otorga pagos o bonos extras; sin embargo, si no aceptan trabajar los fines de semana les sancionan, les bloquean, les bajan el rating y ganan mucho menos dinero por pedido (Página/12, 18/10/2018).

Otra nota periodística permite recuperar, en palabras del abogado de la APP, José Tribuzio, que la capacidad de lucha la Asociación «está en un estado de latencia, con un muy acotado margen de acción. En nuestro caso puntual, cuando creamos 
en 2018 el sindicato APP, las plataformas echaron a toda la comisión directiva», (Risso, 28/07/2020). Tribuzio explica que estas empresas comparten sus formas de inserción en el mercado, el origen externo de sus capitales, las modalidades de organizar el trabajo así como las formas de explotación y de precarización de les trabajadores. "Con esta estrategia de que no hay empleador ni empleados, se implantaron en el mundo al margen de toda legalidad no solo laboral, sino fiscal y tributaria», agrega Tribuzio (Risso, 28/07/2020). Durante el tiempo transcurrido desde el inicio de la cuarentena, han muerto seis repartidores por accidentes fatales. Los modos de ultra precarización depositan en quienes trabajan todos los riesgos: les jóvenes desarrollan el trabajo sin protección, sin cobertura y, como se indicó, todos los gastos corren por su cuenta.

En cuanto a la ley que fue aprobada, dado que la normativa habilita a las empresas como intermediarias de oferta y demanda estas quedan eximidas del cumplimiento de toda normativa laboral y tributaria. Desconocer la figura de la empresa implica, entonces, una desregulación de la economía de las plataformas. Más allá de los beneficios incorporados a favor de les trabajadores -como la obligatoriedad de entregarles los elementos de seguridad e higiene que requieren los cuidados en el marco de las medidas de prevención decretadas por el Estado- la Ley supone la legitimación de los procesos de precarización laboral. El rechazo que obtuvo denuncia el avance de la desregulación de las aplicaciones y las exigencias de obtener un permiso de trabajo que no se les impone ni a otres trabajadores ni a otros servicios. De esta manera, el incumplimiento de no contar con la habilitación tendrá duras sanciones, al punto de poner en riesgo la continuidad en las tareas laborales con estas empresas.

Indiscutiblemente, la creación de la APP, en 2018, las convocatorias a los paros internacionales así como las movilizaciones, las concentraciones y las marchas en sus vehículos de trabajo permiten identificar, como menciona Maristella Svampa (2011), una conexión de les jóvenes con la política. Emerge un ethos de militancia juvenil ligado a matrices político-ideológicas autonomistas y de izquierda, caracterizadas por nuevas formas de organización y por repertorios de acción colectiva. Ampliando esta idea, Paula Varela (2009) coloca en les jóvenes un nuevo sindicalismo, diferenciándolo de un apoliticismo en los años noventa y que tiene, no solo el componente juvenil sino la politización, la acción como herramienta de lucha, el anti-burocratismo y el asambleísmo. 
Desde que las plataformas arribaron a la Argentina la organización colectiva de les trabajadores ha logrado instalar una demanda creciente: el reconocimiento de los derechos laborales de les repartidores de plataformas. La APP como organización incluye la participación de trabajadores autoconvocades, de sindicatos e, incluso, de asociaciones internacionales gremiales tales como la Confederación Sindical de trabajadoras y trabajadores de las Américas y la UNI Global Unión.

Las palabras de Marcos, uno de los siete delegades del personal de plataformas nucleados en la Asociación Sindical de Motociclistas Mensajeros y Servicios (ASIMM), entrevistado por la Agencia Paco Urondo, permite comprender los procesos de construcción de una identidad colectiva frente a estas nuevas formas de empleo, sus demandas y las herramientas para defender sus derechos.

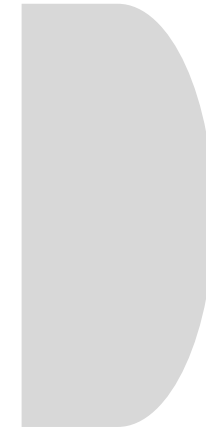

En noviembre (2018), empezaron a llegar algunas cartas documento, ahí hubo entre 200 y 300 despidos. Para diciembre, volvieron a haber otros 200 despidos. Ya para ese entonces estábamos encaminados con el tema del sindicato. [...] le notificamos a la empresa PedidosYA que iba a haber una votación de delegados, prevista para el 23 de febrero. No obstante, esto es como el ajedrez, la empresa agarró y previo a la votación despidió a 250 trabajadores más (Allende \& Carrizo, 23/04/2019).

En cuanto a los obstáculos en el proceso de organización, Marcos señala:

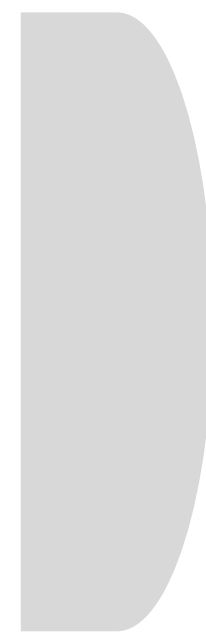

Ellos tienen el poder de manejar el sistema. Lo que vos ficharías de horario en una empresa, ellos lo manejan desde una computadora. [...] los tipos han creado falsas causas para poder pagar menos liquidaciones. Los de PedidosYA adeudan la plata de la nafta desde diciembre [...]. Viene una empresa, te toma como monotributista, no te da aportes, cargas sociales, aguinaldo, vacaciones. Vos estás trabajando, entonces, para un empresario de otro país que viene y se lleva la plata y al trabajador no le da nada. [...] El tema de la caja, que no la pueden llevar en la espalda. Si vos llevás mucho peso estás incómodo, tanto en la bicicleta como en la moto (Allende \& Carrizo, 23/04/2019).

En conclusión, la organización y la identidad colectiva de les trabajadores es muy reciente. Las muertes y los accidentes fatales que sufren muches jóvenes pusieron de relieve la discusión sobre las nuevas formas de explotación del trabajo que el 
neoliberalismo como matriz económica, política e ideológica propone para las juventudes. Frente al «sálvese quien pueda», les jóvenes despliegan una solidaridad afectiva que, para Svampa (2000), implica un cambio de paradigma que las generaciones mayores no entienden, no encuentran esa dimensión expresiva y las formas de solidaridad como corolario en formas de solidaridad laboral. Sin embargo, pocos años de organización no permiten cristalizar identidades que se vinculen o no a tendencias fragmentarias y volátiles (Svampa, 2000). De todas maneras, es preciso subrayar que los procesos se encastran en una matriz conflictiva de relaciones sociales. La apropiación casi absoluta del producto del trabajo de les jóvenes por parte de las empresas patronales y el desprecio por sus vidas derivó en procesos organizativos colectivos donde se sienten, se escuchan, se acompañan y se organizan para defender sus derechos.

\section{Algunas consideraciones finales}

En el presente trabajo se evidenció que en los últimos años la llamada «economía de las plataformas» está cambiando la naturaleza del trabajo en la Argentina y en el mundo. La precarización laboral es un proceso que se agudizó, en términos generales, a partir de las transformaciones en el mundo del trabajo durante los años setenta y constituye uno de los principales problemas que afecta al conjunto de les trabajadores. El caso aquí analizado, permitió la aproximación a diferentes situaciones que hacen posible identificar el deterioro general de las condiciones laborales de les trabajadores de las plataformas, como también visibilizar un conjunto de jóvenes que en el marco de sus jornadas laborales terminan expuestes a riesgos y a accidentes fatales.

El acceso al empleo en las plataformas pone de manifiesto una tendencia de jóvenes calificados que ingresan al mercado de trabajo a través de empleos precarios, teniendo que contar para esto con los recursos necesarios para el desarrollo de las tareas laborales. Les trabajadores expresan posibilidades y expectativas como también elementos relativos al trabajo realizado, sobre las condiciones en las que lo desarrollan y los riesgos a los que se exponen; abanico que nos permite explicar las implicancias de la precariedad laboral más allá de una relación contractual. 
En efecto, el proceso de deterioro de las condiciones materiales y subjetivas implicó experiencias que, a su vez, plantean prácticas. El rechazo de todes les trabajadores a la ley que regula sus tareas señala, con un imperativo de denuncia, la modificación única del Código de Tránsito y Transporte de la Ciudad de Buenos Aires, centrándose en la seguridad vial y en la eliminación de los sistemas de incentivos de las empresas; arbitrariedades que se llevan puesta la vida de jóvenes trabajadores.

Asimismo, el trabajo en las plataformas no está exento de las desigualdades de género presentes en nuestras sociedades. La inequidad en el acceso para las jóvenes como una parte del conjunto de desigualdades que las atraviesan «se explica por la discriminación que realizan las empresas al momento de la contratación, pero otras preexisten al momento de la inserción laboral» (Pérez, 2016, p. 48). Las trabajadoras y el colectivo LGBT+ son les más perjudicades: no cuentan con licencias por maternidad, no tienen garantizados los lugares de aseo, de descanso ni locaciones con baños. El acoso callejero, los robos y los accidentes de tránsito son moneda corriente en el marco de sus jornadas laborales.

El escenario de la pandemia reconfiguró los diferentes ámbitos de la vida de millones de argentines. Es necesario readecuar normativas y establecer marcos legales que brinden herramientas de protección legal a les trabajadores, para mejorar los aspectos de la seguridad vial y las medidas de protección. El presente trabajo abordó la situación de precariedad laboral de les trabajadores desde una mirada histórica, contextualizada en el marco de relaciones económicas, sociales, culturales y que coloca a les trabajadores, sus experiencias, sus expectativas y sus sentires en el centro del análisis. Esto permite generar aproximaciones para la construcción de explicaciones sobre el proceso de deterioro de sus condiciones de trabajo, tanto materiales como subjetivas.

Los procesos de precarización laboral son temas emblemáticos en la organización y en la movilización social. Hasta aquí, intentamos simplificar, en muy pocas palabras, un proceso cargado de historias, tensiones, organización, disputas de sentidos, proyectos, que exige revisar condiciones económicas, sociales y políticas tanto locales como nacionales e internacionales. Este proceso se ubica en el marco general de las tensiones, los intereses contradictorios y las luchas presentes en las relaciones sociales de nuestras sociedades. Resulta pertinente e interesante 
analizar el contexto de oportunidad política que se abre con el gobierno actual en una de las sociedades tradicionalmente más movilizadas de la región. En el caso de les trabajadores de las plataformas, convergen una serie de problemáticas estructurales y emergentes, puesto que se inscribe en una tendencial desregulación laboral como producto del desarrollo capitalista y de las formas de precarización imperantes desde la década del setenta. Entre las cuestiones emergentes, uno de los aspectos que podría ser objeto de ulteriores estudios es el de los repertorios de acción colectiva que despliegan les trabajadores de estas plataformas y las coaliciones que se configuran en la acción contenciosa. En ese sentido, huelga indagar el grado de representatividad que asumen estas recientes organizaciones y sus desafíos para la representación colectiva.

Finalmente, resulta imprescindible avanzar en la regulación del trabajo en las plataformas. No podemos pensar las empresas como intermediarias entre la oferta y la demanda, lo que consecuentemente implique una des-responsabilidad de estas sobre las condiciones laborales de les trabajadores. La necesidad de regulación, a su vez, no implica la urgencia o la inmediatez; debemos recordar que la reforma laboral se instaló en el país durante los últimos años y aún sigue latente. Por lo tanto, para evitar las regulaciones que crean este tipo de empleos, sobre estos procesos de precariedad laboral y de leyes flexibilizadoras, se requiere de un debate delicado que asuma el análisis de los factores económicos, políticos, sociales y culturales del mercado de trabajo. Así, hablar del mundo del trabajo contrarresta tendencias que reducen la mirada a la compra y a la venta de fuerza de trabajo, y permite ubicar el trabajo como actividad humana de transformación social, del contexto y de les jóvenes trabajadores para construir los marcos legislativos laborales para sociedades más justas y más humanas.

\section{Referencias}

Allende, M. S. y Carrizo, P. (23 de abril de 2019). Trabajadoras y trabajadores de aplicaciones se organizan contra la precarización. Agencia Paco Urondo. Periodismo militante. Recuperado de https://www.agenciapacourondo.com.ar/sindicales/lasostrabajadorases-de-aplicaciones-se-organizan-contra-la-precarizacion 
Contra la ley de apps (18 de julio de 2020). Página/12. Recuperado de https://www.pagina12.com.ar/279221-contra-la-ley-de-apps

De Gennaro, L. (27 de julio de 2020). Precario es el mundo. Sangrre. Recuperado de https://sangrre.com.ar/2020/07/27/precario-es-elmundo/

Diana Menéndez, N. (2010). La múltiple dimensión de la precariedad laboral: el caso de la administración pública en Argentina. Revista de Ciencias Sociales, II-IIl(128-129), 119-136. https://doi.org/10.15517/rcs.v0i128-129.8760

Fernández de Kirchner, C. (noviembre de 2018). Capitalismo, neoliberalismo y crisis de la democracia [conferencia]. Primer Foro Mundial del Pensamiento Crítico organizado por el Consejo Latinoamericano de Ciencias Sociales, Argentina. Recuperado de https://clacso.org.ar/clacsotv/conferencias_foros_debates.php?id_vide $0=1133$

INDEC. (2011). Encuesta Permanente de Hogares. Conceptos de condición de actividad, subocupación horaria y categoría ocupacional. Ministerio de Economía y Finanzas Públicas, Buenos Aires.

Página/12 (11 de octubre de 2018). La APP contra la precarización. Recuperado de https://www.pagina12.com.ar/147845-la-app-contra-laprecarizacion

Ley 6.314 (2020). Regulación de las tareas laborales. Ciudad Autónoma de Buenos Aires, Argentina. Recuperado de http://www2.cedom.gov.ar/es/legislacion/normas/leyes/ley6314.html

Longo, J. y Busso, M. (2017). Precariedades. Sus heterogeneidades e implicancias en el empleo de los jóvenes en Argentina. Estudios del Trabajo, (53). Recuperado de https://aset.org.ar/ojs/revista/article/view/6 
Madariaga, J., Buenadicha, C., Molina, E. y Ernst, C. (2019). Economía de plataformas y empleo ¿Cómo es trabajar para una app en Argentina? CIPPEC-BID-OIT. Recuperado de https://www.cippec.org/publicacion/economia-de-plataformas-yempleo-como-es-trabajar-para-una-app-en-argentina/

Gestión Sindical (10 de octubre de 2018). Nace una app para luchar contra la precarización laboral. Recuperado de https://gestionsindical.com/nace-una-app-para-luchar-contra-laprecarizacion-laboral/

Pérez, P. (2016). Jóvenes, trabajo y desigualdades de género en Argentina (2003-2014). En M. Busso y P. Pérez, Caminos al trabajo: el mundo laboral de los jóvenes durante la última etapa del gobierno kirchnerista (pp. 33-50). Ciudad Autónoma de Buenos Aires, Argentina: Miño y Dávila.

Risso, N. (28 de julio de 2020). El método Glovo, Rappi y PedidosYA. Página/12. Recuperado de https://www.pagina12.com.ar/281256-elmetodo-glovo-rappi-y-pedidos-ya

Svampa, M. (2000). Desde abajo: la transformación de las identidades sociales. Ciudad Autónoma de Buenos Aires, Argentina: Biblos.

Svampa, M. (2011). Argentina una década después. Del «que se vayan todos» a la exacerbación de lo nacional-popular. Nueva Sociedad, (235), 17-34. Recuperado de https://nuso.org/articulo/argentina-una-decadadespues-del-que-se-vayan-todos-a-la-exacerbacion-de-lo-nacionalpopular/

Varela, P. (2009). ¿De dónde salieron estos pibes? Consideraciones sobre el activismo gremial de base en Argentina posdevaluación. Margen. Revista Margen de Trabajo Social y Ciencias Sociales, (55), 1-14. Recuperado de http://www.margen.org/suscri/margen55/varela.pdf 


\section{Notas}

1 En el trabajo se sintetizan algunas reflexiones iniciadas en el marco de seminario «óvenes, trabajo y precarización laboral», dictado durante el primer cuatrimestre del corriente año por la Dra. Marina Adamini y por el Dr. Daniel Dicósimo para las carreras Licenciatura en Trabajo Social y Licenciatura en Historia de la Facultad de Ciencias Humanas de la Universidad Nacional del Centro de la Provincia de Buenos Aires (UNICEN).

2 El Aislamiento Social Preventivo y Obligatorio (ASPO) es una medida excepcional adoptada por el Gobierno argentino, mediante el Decreto 297/2020, ante el brote de coronavirus declarado como Pandemia por la Organización Mundial de la Salud (OMS).

3 Desde una perspectiva de género en la construcción del lenguaje inclusivo que problematiza el discurso y la lingüística, la utilización de la «e» consiste en suprimir el morfema de género que conlleva la utilización de las vocales «a» y «0» para incluir en la semántica de la palabra cualquier género o persona.

4 El Centro de Implementación de Políticas Públicas para la Equidad y el Crecimiento (CIPPEC) es una organización independiente, apartidaria y sin fines de lucro que produce conocimiento y ofrece recomendaciones para construir mejores políticas públicas.

5 Quedan invisibilizadas dentro de la Población Económicamente Activa (PEA), categoría en la que el INDEC, partiendo del concepto de trabajo entendido como una actividad que genera bienes o servicios para el mercado, considera ocupadas a todas las personas que tengan cierta edad especificada (10 años o más) y que durante un período de referencia (una semana) hayan trabajado al menos una hora. Se incluyen en esta categoría: personas que durante el período de referencia realizaron algún trabajo de al menos una hora, hayan recibido pago (en dinero o en especie) o no por dicha actividad; y personas que tienen una ocupación pero que no estaban trabajando temporalmente durante el período de referencia y mantenían un vínculo formal con su empleo. 